\title{
Prevalence of Adult Attention Deficit Hyperactivity Disorder and its Correlates among Recently Diagnosed Young Adult Males with HIV
}

Shankar Kumar ${ }^{1 *}$, Aashik Shekar ${ }^{1}$, Kasthuri Pandiyan ${ }^{1}$, Gopal Das ${ }^{1}$, Abhinav Nahar ${ }^{1}$, Kodur Ramamurthy Raveendra ${ }^{2}$ and Chandrashekar Hongally ${ }^{1}$

${ }^{1}$ Department of Psychiatry, Bangalore Medical College and Research Institute, Bangalore, India

${ }^{2}$ Department of Internal medicine, Bangalore Medical College and Research Institute, Bangalore, India

\begin{abstract}
A majority of children with symptoms of Attention Deficit Hyperactivity Disorder (ADHD) from early childhood continue to demonstrate notable ADHD symptoms throughout life. ADHD-associated impulsivity, in adults, has been found to be a predictor of unstable interpersonal relationships and high risk sexual behavior. This study was undertaken to determine the prevalence of adult ADHD in recently diagnosed young male HIV patients.

100 young adult males who were diagnosed with HIV less than a year ago were recruited after an informed consent and were administered the following questionnaires-socio-demographic questionnaire with details of HIV diagnosis, HIV High Risk Behavior Questionnaire (HRBS) to measure High risk sexual behaviors, MINI neuropsychiatric Interview 6.0 to rule out psychiatric co-morbidities, Mini Mental State Examination (MMSE) to rule out major cognitive impairment, Adult ADHD Self Report scale (ASRS) v1.1 to screen for ADHD in adulthood. ADHD-rating scale was administered to parent/ informant to screen for childhood ADHD. Statistical analyses were done using SPSS v 17.0.

The prevalence of Adult ADHD in our sample was $20 \%$. 94\% of subjects were on Highly Active Anti-Retroviral Therapy (HAART). Adherence to HAART was comparatively lesser in the ADHD group which was statistically significant $(p=0.014)$. Substance use was significantly more in the ADHD group $(p=0.03)$. People with ADHD had significantly higher High Risk sexual behavior than those without ADHD $(p<0.001)$. Hence, these findings suggest that ADHD may contribute to high risk sexual behavior and thereby also the risk of contracting HIV. Future research on a larger population will provide more conclusive evidence. For now, health promotion and specific protection with greater emphasis in this vulnerable population of adult ADHD would be warranted in preventing HIV.
\end{abstract}

Keywords: Attention Deficit Hyperactivity Disorder; HIV; Sexual behavior; Highly Active Antiretroviral Therapy; Antisocial personality disorder

\section{Introduction}

Attention deficit-hyperactivity disorder [ADHD] is a neurobehavioral disorder characterized by either significant difficulties of inattention or hyperactivity and impulsivity or a combination of the two. $15-20 \%$ of children with ADHD maintain the full diagnosis and as many as $30-60 \%$ of these will maintain the full diagnosis or some residual symptoms as adults [1,2]. The prevalence rate of ADHD in the adult population in some continents is $4-5 \%[3,4]$.

This may manifest as multiple problems based on the age of the individual. The core difficulties in executive functioning seen in ADHD [5] result in a different picture in later life, depending upon the demands made on the individual by their environment. Individuals during adolescence may manifest with risky driving which includes lethal Road Traffic accidents [6]. It may also manifest with early onset of severe drug and substance dependence [7]. They may also develop a distorted sense of self and disruption of development of self in adolescence [8]. Later in life, ADHD can manifest with multiple dismissals from employment, difficulties in work place such as interpersonal and relationship difficulties, multiple absenteeism and excessive errors [9]. There is very limited evidence to suggest that ADHD could contribute to risky sexual behavior in early adulthood [10].

The incidence of HIV is increasing at alarming proportions and one of the main factors for it being high risk sexual behavior. As preliminary findings have suggested, ADHD in adulthood could contribute to high risk sexual behavior [11] and this, being a potentially treatable condition, could lead us to newer primary prevention strategies for HIV care.

\section{The hypothesis}

Adult ADHD is associated with increased prevalence of early high risk sexual behaviors, in this population with HIV.

\section{Aims and Objectives}

To estimate the prevalence of Adult ADHD in young adult males, who were diagnosed of HIV less than a year ago.

\section{Methodology}

Study design- Cross sectional prevalence study. This study was carried out in the Anti-Retroviral Therapy (ART) Centre-a nodal centre for management of people living with HIV in Karnataka state and the Department of Psychiatry, Victoria Hospital, which is a postgraduate teaching hospital attached to Bangalore Medical College. Bangalore is a metropolitan city and the capital of Karnataka state (India) with

*Corresponding author: Shankar Kumar, Department of Psychiatry, Bangalore Medical College and Research Institute, Bangalore, Tel: 919844546083; E-mail: shankarkjs@gmail.com

Received April 23, 2014; Accepted May 26, 2014; Published June 10, 2014

Citation: Kumar S, Shekar A, Das G, Nahar A, Pandiyan K, et al. (2014) Prevalence of Adult Attention Deficit Hyperactivity Disorder and its Correlates among Recently Diagnosed Young Adult Males with HIV. J AIDS Clin Res 5: 314. doi:10.4172/21556113.1000314

Copyright: (C) 2014 Kumar S, et al. This is an open-access article distributed under the terms of the Creative Commons Attribution License, which permits unrestricted use, distribution, and reproduction in any medium, provided the original author and source are credited. 
a population of around 10 million. Around 80-100 new patients are diagnosed with HIV and 200-250 patients receive HAART every month in the ART centre under the National AIDS control program (NACO), India.

The duration of study was 2 months and we could get 100 patients during this period who consented for the study. These 100 consecutive young adult males, aged 20-35 years, who were diagnosed of HIV infection within the past year, were recruited from the ART center of Victoria hospital after taking informed written consent from the participants.

Patients who were already diagnosed with major Axis-1 psychiatric disorders, Intellectual disability, those on psychiatric management, those with a score of less than 23 on Mini Mental Status Examination and patients for whom a family member for corroboration of Diagnosis of childhood ADHD could not be obtained, were excluded from the study.

For all recruited subjects, a Socio-demographic questionnaire which assessed relevant socio-demographic details, details about diagnosis of HIV, CD 4 counts, intake of ART, medical co morbidities, use of alcohol and various substances of abuse was administered. The subjects were administered the HIV Risk taking Behavior Scale (HRBS) which is a 11 item scale that measures aspects of Injection Drug use (IDU) and High risk Sexual behavior among subjects [12]. They were then administered the MINI Neuropsychiatric Interview 6.0 [13] to rule out major Psychiatric co-morbidities as used in recent studies [14] and to look for a categorical diagnosis of Anti-social personality disorder and Mini Mental State Examination to rule out Major Cognitive Impairment.

They were then administered Adult ADHD self-report scale [ASRS v1.1] to screen for Adult ADHD [15]. The Symptom Checklist is an instrument consisting of the eighteen DSM-IV-Text Revision (TR) criteria for ADHD in adulthood. Six of the eighteen questions were found to be the most predictive of symptoms consistent with ADHD. One family member of the subject was administered ADHD rating scale for measuring Childhood ADHD in the patient which diagnoses ADHD as inattentive/ Impulsive and Hyperactivity/ Combined subtypes based on DSM-4 criteria.

10 subjects who could not bring informants and 7 who did not consent were excluded from the study. Statistical analysis was done using unpaired t-test and chi-square test and results were tabulated.

\section{Results}

The prevalence of Adult ADHD in the sample was 20\% $(n=20)$ (Table 1-4 and Figure 1 and 2).

\section{Discussion}

The aim of the study was to find the prevalence of Adult ADHD in recently diagnosed young adult males with HIV. We included only recently diagnosed individuals with HIV as bias due to cognitive impairment could be minimized. The population studied was young adult males with HIV because the hypothesis was that, those with childhood ADHD, which is higher in males, would have high risk sexual behavior which would predispose them to HIV early in life.

The prevalence of Adult ADHD in this study is $20 \%$, which is

\begin{tabular}{|c|c|c|c|}
\hline & Total Sample & ADHD group & Non-ADHD group \\
\hline Age(years) & $31.2 \pm 3.5$ & $31.1 \pm 3.5$ & $30.7 \pm 3.4$ \\
\hline Marital status n (\%) & $69(69 \%)$ & $54(67.5 \%)$ & $15(75 \%)$ \\
\hline
\end{tabular}

Table 1: Socio-demographic variables.

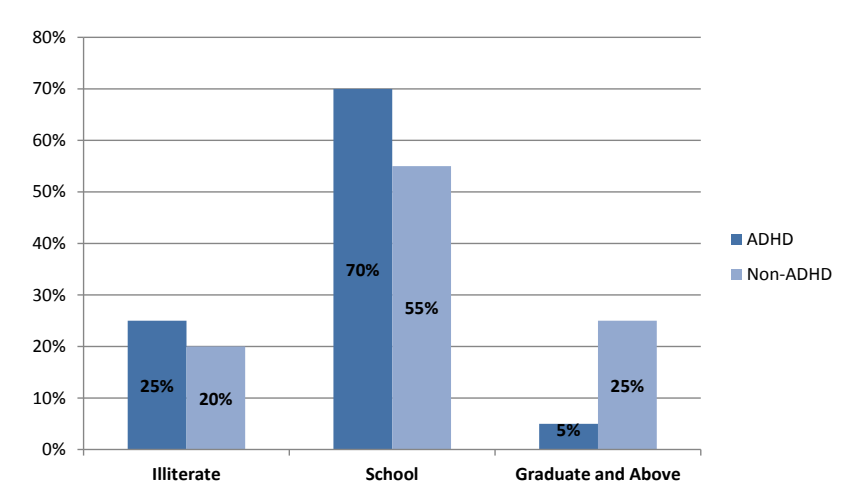

Note- A majority of those with ADHD (95\%) could not complete graduation whereas $25 \%$ of those without ADHD were graduates.

Figure 1: Educational status of ADHD and Non-ADHD groups.

\begin{tabular}{|c|c|c|c|c|c|c|c|c|c|}
\hline & \multicolumn{2}{|c|}{ Total sample } & \multicolumn{2}{|c|}{ ADHD group } & \multicolumn{2}{|c|}{$\begin{array}{c}\text { Non-ADHD } \\
\text { group }\end{array}$} & $\mathbf{p}$ & $\mathbf{d f}$ & $\mathbf{t}$ \\
\cline { 2 - 9 } & Mean & SD & Mean & SD & Mean & SD & & \\
\hline $\begin{array}{c}\text { HIV duration } \\
\text { (months) }\end{array}$ & 8.9 & 3.9 & 7.55 & \pm 3.59 & 9.39 & \pm 3.25 & $\mathbf{0 . 0 2 9 7 ^ { * }}$ & 95 & 2.2077 \\
\hline CD 4 counts & 342.6 & 196.4 & 399.1 & $\begin{array}{l} \pm \\
270.92\end{array}$ & 329.2 & \pm 175.61 & 0.160 & 98 & 1.4139 \\
\hline $\begin{array}{c}\text { Individuals } \\
\text { on HAART }\end{array}$ & 94 & $94 \%$ & 17 & $85 \%$ & 77 & $96.25 \%$ & $\mathbf{0 . 0 1 7 1 *}$ & 1 & 1.873 \\
\hline $\begin{array}{c}\text { Good } \\
\text { Adherence }\end{array}$ & 82 & $87.2 \%$ & 11 & $64.7 \%$ & 70 & $90.9 \%$ & $\mathbf{0 . 0 1 4}$ & 1 & 5.975 \\
\hline
\end{tabular}

Note: $S D=$ standard deviation, $p=$ probability value for the t-test or chi-square test, $\mathrm{df}=$ degrees of freedom, $\mathrm{t}=\mathrm{t}$ value for the two tailed $\mathrm{t}$-test, $\mathrm{x} 2=$ chi square value for chi-square test. * indicates statistical significance at $p<0.05$. Good adherence was defined as a minimum of $95 \%$ intake of prescribed HAART therapy.

Table 2: Comparison of HIV related variables between ADHD and Non - ADHD groups.

\begin{tabular}{|c|c|c|c|c|c|}
\hline & $\begin{array}{c}\text { ADHD Group } \\
\mathbf{n}(\%)\end{array}$ & $\begin{array}{c}\text { Non ADHD } \\
\text { Group n (\%) }\end{array}$ & P & df & X2 \\
\hline $\begin{array}{c}\text { Presence of } \\
\text { substance use }\end{array}$ & $19(95 \%)$ & $55(68.7 \%)$ & $\mathbf{0 . 0 3}^{*}$ & 1 & 4.447 \\
\hline $\begin{array}{c}\text { Suicidal Attempts } \\
\text { Co-morbid ASPD }\end{array}$ & $5(25 \%)$ & $15(18.7 \%)$ & 0.754 & 1 & 0.098 \\
\hline
\end{tabular}

Note: Sample size of $A D H D$ group $=20$, non $A D H D=80$. $p=$ probability value for chi square test. ${ }^{*}$ indicates statistical significance at $p<0.05$. $d f=$ degrees of freedom. $\mathrm{x}^{2}=$ chi square value.

Table 3: Association between substance use, suicidal attempts and presence of co-morbid ASPD between ADHD and Non-ADHD groups.

\begin{tabular}{|c|c|c|c|c|c|c|c|}
\hline & \multicolumn{2}{|c|}{ ADHD Group } & \multicolumn{2}{|c|}{ non-ADHD Group } & \multirow{2}{*}{$\mathbf{P}$} & \multirow{2}{*}{$\mathbf{T}$} & \multirow{2}{*}{ df } \\
\hline & Mean & SD & Mean & $\mathrm{SD}$ & & & \\
\hline Mean HRBS score & 4.75 & 3.34 & 1.01 & 0.92 & $<0.001^{*}$ & 8.72 & 95 \\
\hline
\end{tabular}

Note: $S D=S t a n d a r d$ deviation. $p=$ probability value for the t-test. $D f=$ degrees of freedom. ${ }^{*}$ indicates statistical significance at $p<0.05$. Sample size of $A D H D=20$, non $\mathrm{ADHD}=80$.

Table 4: Comparison of high risk sexual behavior between ADHD and Non-ADHD groups.

4-5 times higher than the prevalence rate of ADHD in the adult general population suggesting that $\mathrm{ADHD}$ is over-represented in this population with HIV $[2,3]$.

\section{Educational levels}

In our study population, we found that a majority of those with 


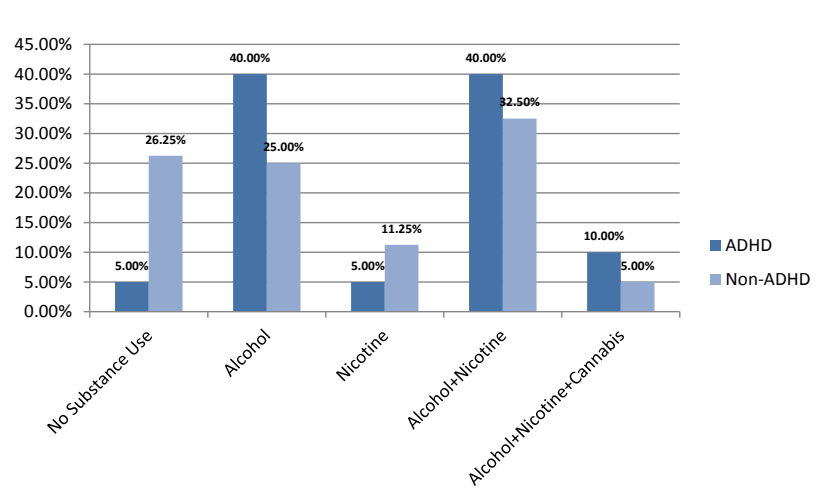

Note: Values shown are in percentage. There were 19 individuals with ADHD (out of 20 ) and 55 individuals without ADHD (out of 80 ) with substance use. Majority of those with ADHD had either alcohol/ alcohol with nicotine use (40\%). A quarter of those without ADHD had no substance use $(26.25 \%)$ whereas only $5 \%$ of those with ADHD had no substance use.

Figure 2: Substance use in ADHD and Non-ADHD groups.

ADHD had only formal school education $(n=70)$ and a very small minority could complete graduation or beyond $(n=5)$ in contrast to those without ADHD wherein a quarter of the population could complete graduation or beyond $(n=25)$. Presence of Inattention and or Hyperactivity and impulsivity in childhood is associated with poor academic outcomes [16]. Hence, in our study population, those with ADHD could probably complete only formal school education and could not pursue higher education due to persisting symptomatology. Moreover, none of those with ADHD were on treatment.

\section{HIV treatment variables}

94 subjects interviewed were on Anti-Retroviral Therapy (94\%). This means that a majority of our sample were already on ART in spite of them being detected with HIV infection a mean of just 8.9 months prior to assessment. This could be possibly because the setup is a tertiary referral /nodal center for HIV care with free treatment being provided under the National AIDS Control Programme in India. Thus, those with diagnosed HIV are referred for ART from other centers which could explain the high numbers. Significantly more number of individuals without ADHD were on ART than those with ADHD $(p=0.017)$. This could possibly be explained by longer HIV duration in those without ADHD than with the disorder $(\mathrm{p}=0.02)$ which was seen in the sample.

Individuals with ADHD were less likely to be adherent to ART than those without ADHD ( $p=0.014)$. Adherence in our study was defined as intake of a minimum of $95 \%$ of pills which were expected to be taken by the person with HIV, which is as defined by National AIDS Control Organization, India [17]. As per existing literature, HIV associated neurocognitive deficits increase the risk of non-adherence in HIV. Neurocognitive deficits in HIV may arise due to the disease process itself, as a result of co morbid substance use, associated psychiatric illnesses such as bipolar disorders, schizophrenia and developmental disorders such as ADHD and conduct disorder [18]. Cognitive deficits in ADHD being potentially treatable, may offer a new strategy for enhancing treatment adherence in HIV.

\section{Substance use}

People with ADHD had higher prevalence of substance use than the non-ADHD group which again showed statistical significance $(\mathrm{p}=0.03)$. The findings are in concordance with existing literature, which show that persisting hyperactivity/impulsivity has a pronounced risk of early onset substance use [19]. Research has also demonstrated preliminary evidence of a protective effect of stimulant therapy (for treatment of ADHD) on later development of severe substance use disorders [20]. Traditionally, though stimulants are believed to be associated with a high abuse potential, studies have demonstrated that use of extended release formulations and formulations such as Lisdexamphetamine, are not associated with increased abuse potential [21].

This has important implications because substance use itself is a risk factor for HIV [22] and presence of ADHD may be a compounding factor, which needs to be further evaluated in future research. Also, treatment strategies early in life for ADHD could potentially decrease risk of future substance use which may have preventive implications for HIV.

Majority (95\%) of those with ADHD had alcohol/ both alcohol and nicotine use. None of them reported Injection Drug Use (IDU). This could reflect cultural patterns of substance use, as IDU is relatively rare in India as compared to the west, while alcohol and nicotine are the most commonly used substances [23]. It is also interesting to note that none of those with ADHD $(n=20)$ were diagnosed with the disorder previously and hence, none were on stimulants.

\section{Suicide}

There were no statistically significant differences when rates of suicide attempts were compared between those with and without ADHD ( $\mathrm{p}=0.754)$. We had hypothesized that suicide attempts would be higher in those with ADHD due to high impulsivity. However, we could not find this. The possible reason for this could be explained by the fact that suicidal behavior could also be due to other risk factors such as high risk personality traits (like impulsivity in Borderline personality) [24], mood disorders [25] which were not evaluated in the study.

\section{Co-morbidity with Anti-Social Personality Disorder}

Out of the 20 subjects diagnosed with Adult ADHD, 45\% were also diagnosed with ASPD using MINI 6.0. This suggests that either ADHD is highly co morbid with ASPD [26] or it could also suggest that these disorders need to be carefully delineated as impulsivity forms a common domain between both the disorders. Presence of ASPD predicts poor response to treatment strategies and poor outcome [27] and these individuals could need long term psychotherapeutic care in addition to other treatment modalities.

\section{High-risk sexual behavior}

There was significantly higher prevalence of high-risk sexual behavior in the preceding month among those with ADHD than those without ADHD ( $\mathrm{p}<0.0001)$. In earlier research, Kate Flory [9] and colleagues also suggested that ADHD is a potential risk factor for high risk sexual behavior which could be mediated by factors such as interpersonal factors, peer relationships, family factors and most importantly, core features of the disorder such as impulsivity itself.

Though the mean duration of HIV in those with ADHD was 7.55 months, a significant majority of them indulged in high risk sexual behavior in the preceding month, though they were aware of their clinical status of HIV. This indicates that ADHD is probably a potential risk factor for continued high risk sexual behavior in HIV. It needs to be studied in future if treatment directed at ADHD could possibly prevent continued high risk sexual behavior. This may have important implications for HIV preventive research. 
Majority (45\%) of those with ADHD in our study also had co morbid Anti-social Personality disorder which could add to the risk of high- risk sexual behavior as evident in previous research [28].

\section{Conclusion}

The prevalence of Adult ADHD in our population of recently diagnosed young adult males with HIV is $20 \%$, which is $4-5$ times that of the general population. People with ADHD had higher prevalence of high-risk sexual behavior and substance use, even after HIV was diagnosed. This makes it an important area for planning primary prevention strategies in HIV care.

\section{Limitations}

a) Relatively small sample size

b) Population from a single clinic, which limits generalizability

c) The population surveyed in this study is unlike those in other studies mentioned in terms of geographic catchment area.

d) ADHD was diagnosed clinically and using ASRS, but needs confirmation with reliable scales such as Wender-Reimherr Adult ADHD Rating Scale.(WRAADHDRS) [29].

e) A categorical diagnosis of Anti-social Personality disorder was assessed but traits were not evaluated. Impulsivity as a trait is more important to assess as this could mediate effects of ADHD and sexual behavior.

f) Substance use was measured, but assessments of severity were not done.

g) Confounding factors like cognitive impairment due to HIV itself were measured by MMSE, but more reliable neurocognitive assessments could have been used.

h) Though an association between ADHD and HIV was established, factors mediating the association, possibly conduct/ASPD traits or presence of substance use could have been ascertained.

\section{Future directions}

Though it can be presumed that impulsivity and high risk sexual behavior mediates the association between ADHD and HIV, the directionality of association needs to be studied. Further studies need to be done with longitudinal assessments after treatment of ADHD to establish the potential importance of management of ADHD as a primary prevention target strategy for HIV in this vulnerable population.

\section{Acknowledgement}

Karnataka State AIDS Prevention Society, Bangalore for permitting us to conduct the study at Anti Retroviral Therapy centre, Victoria Hospital and Indian council of medical research, STS programme 2013.

\section{References}

1. Weiss G, Hechtman LT (1993) Hyperactive children grown up: ADHD in children, adolescents and adults. Guildford, New York.

2. Willoughby MT (2003) Developmental course of ADHD symptomatology during the transition from childhood to adolescence: a review with recommendations. J Child Psychol Psychiatry 44: 88-106.

3. Goodman DW, Thase ME (2009) Recognizing ADHD in adults with comorbid mood disorders: implications for identification and management. Postgrad Med 121: $20-30$.

4. Kessler RC, Adler L, Barkley R, Biederman J, Conners CK, et al. (2006) The prevalence and correlates of adult ADHD in the United States: results from the National Comorbidity Survey Replication. Am J Psychiatry 163: 716-723.
5. Barkley RA (1997) Behavioral inhibition, sustained attention, and executive functions: constructing a unifying theory of ADHD. Psychol Bull 121: 65-94.

6. Kaya A, Taner Y, Guclu B, Taner E, Kaya Y, et al. (2008) Trauma and adult attention deficit hyperactivity disorder. J Int Med Res 36: 9-16.

7. Ohlmeier MD, Peters K, Kordon A, Seifert J, Wildt BT, et al. (2007) Nicotine and alcohol dependence in patients with comorbid attention-deficit/hyperactivity disorder (ADHD). Alcohol Alcohol 42: 539-543.

8. Krueger M, Kendall J (2001) Descriptions of self: an exploratory study of adolescents with ADHD. J Child Adolesc Psychiatr Nurs 14: 61-72.

9. Mannuzza S, Klein RG, Bessler A, Malloy P, LaPadula M (1993) Adult outcome of hyperactive boys. Educational achievement, occupational rank, and psychiatric status. Arch Gen Psychiatry 50: 565-576.

10. Flory K, Molina BS, Pelham WE Jr, Gnagy E, Smith B (2006) Childhood ADHD predicts risky sexual behavior in young adulthood. J Clin Child Adolesc Psycho 35: 571-577.

11. Galéra C, Messiah A, Melchior M, Chastang JF, Encrenaz G, et al. (2010) Disruptive behaviors and early sexual intercourse: The GAZEL Youth Study. Psychiatry Res 177: 361-363.

12. Darke S, Hall W, Heather N, Ward J, Wodak A (1991) The reliability and validity of a scale to measure HIV risk taking behaviour among intravenous drug users. AIDS 5: 181-185.

13. Williams JB, Spitzer RL, Gibbon M (1992) International reliability of a diagnostic intake procedure for panic disorder. Am J Psychiatry 149: 560-562.

14. Stylianidis S, Pantelidou S2, Chondros P2, Roelandt JL3, Barbato A4 (2014) Prevalence of mental disorders in a Greek island. Psychiatrike 25: 19-26.

15. Kessler RC, Adler L, Ames M, Demler O, Faraone S, et al. (2005) The World Health Organization Adult ADHD Self-Report Scale (ASRS): a short screening scale for use in the general population. Psychol Med 35: 245-256.

16. Washbrook E, Propper C, Sayal K (2013) Pre-school hyperactivity/attention problems and educational outcomes in adolescence: prospective longitudinal study. Br J Psychiatry 203: 265-271.

17. Sahay S, Reddy KS, Dhayarkar S (2011) Optimizing adherence to antiretroviral therapy. Indian J Med Res 134: 835-849.

18. Anand P, Springer SA, Copenhaver MM, Altice FL (2010) Neurocognitive impairment and HIV risk factors: a reciprocal relationship. AIDS Behav 14: 1213-1226.

19. Chang Z, Lichtenstein P, Larsson $H$ (2012) The effects of childhood ADHD symptoms on early-onset substance use: a Swedish twin study. J Abnorm Child Psychol 40: 425-435.

20. Zulauf CA, Sprich SE, Safren SA, Wilens TE (2014) The complicated relationship between attention deficit/hyperactivity disorder and substance use disorders. Curr Psychiatry Rep 16: 436.

21. Faraone SV, Upadhyaya HP (2007) The effect of stimulant treatment for ADHD on later substance abuse and the potential for medication misuse, abuse, and diversion. J Clin Psychiatry 68: e28.

22. Pickens RW, Battjes R, Svikis DS, Gupman AE (1993) Substance use risk factors for HIV infection. Psychiatr Clin North Am 16: 119-125.

23. Murthy P, Manjunatha N, Subodh BN, Chand PK, Benegal V (2010) Substance use and addiction research in India. Indian J Psychiatry 52: S189-199.

24. Brent DA, Johnson BA, Perper J, Connolly J, Bridge J, et al. (1994) Personality disorder, personality traits, impulsive violence, and completed suicide in adolescents. J Am Acad Child Adolesc Psychiatry 33: 1080-1086.

25. Sanchez LE, Le LT (2001) Suicide in mood disorders. Depress Anxiety 14: 177 182.

26. Cumyn L, French L, Hechtman L (2009) Comorbidity in adults with attentiondeficit hyperactivity disorder. Can J Psychiatry 54: 673-683.

27. Brooner RK, Greenfield L, Schmidt CW, Bigelow GE (1993) Antisocial personality disorder and HIV infection among intravenous drug abusers. Am J Psychiatry 150: 53-58.

28. Ramrakha S, Caspi A, Dickson N, Moffitt TE, Paul C (2000) Psychiatric disorders and risky sexual behaviour in young adulthood: cross sectional study in birth cohort. BMJ 321: 263-266.

29. Marchant BK, Reimherr FW, Robison D, Robison RJ, Wender PH (2013) Psychometric properties of the Wender-Reimherr adult attention deficit disorder scale. Psychol Assess 25: 942-950. 